Qualitative-other

\section{Dutch midwives perceive a mixture of rewards and difficulties when caring for non-western women}

10.1136/eb-2014-101736

\section{Maggie Redshaw}

Policy Research Unit for Maternal Health and Care, National Perinatal Epidemiology Unit, Nuffield Department of Population Health, University of Oxford, Oxford, UK

Correspondence to: Dr Maggie Redshaw, Policy Research Unit for Maternal Health and Care, National Perinatal Epidemiology Unit, Nuffield Department of Population Health, University of Oxford, Oxford 0X3 7LF, UK; maggie.redshaw@npeu.ox.ac.uk

Commentary on: Boerleider AW, Francke AL, Manniën J, et al. 'A mixture of positive and negative feelings': a qualitative study of primary care midwives' experiences with non-western clients living in the Netherlands. Int J Nurs Stud 2013;50:1658-66.

\section{Implications for standard and practice}

- This study is valuable for its particular focus on Dutch midwives' experiences of working with and caring for non-western women.

- The findings need to be bolstered by further research on the experiences of non-western women themselves in order to better understand their needs in the context of maternity care.

- Midwives must be proactive and flexible and also need more time and resources to aid communication with non-western clients.

\section{Context}

Migration is a common event, with increasing numbers of migrants living in developed parts of the world. A range of studies have shown that the maternity healthcare needs of migrant women and those from minority ethnic groups are not always met as effectively as those of women in the majority population. ${ }^{1}$

A large proportion of those receiving maternity care from Dutch midwives are of non-western in origin, with at least one parent born in Africa, Asia, South America or Turkey. This group of women has been shown to make less than optimal use of antenatal care, characterised by later initial contact with the care system. Higher rates of perinatal mortality have also been observed among some non-western groups.

Boerleider and colleagues sought to explore Dutch midwives' experiences of treating non-western clients.

\section{Methods}

Using convenience sampling and a semistructured topic guide, qualitative interviews were carried out with 13 midwives, while eight midwives participated in a focus group interview. Thematic analysis was used for handling the data in an iterative way, with subthemes and overarching main themes being developed.

\section{Findings}

Three main themes were identified: 'having a mixture of positive and negative feelings', 'fınding ethnic diversity fascinating, but also quite difficult' and 'aiming for optimal care in the interests of both mother and child'. Caring for non-western women was both rewarding and demanding. Workload, a lack of understanding about the maternity care system and language barriers were frequently mentioned as issues in delivering care to non-western women.

The authors concluded that providing care to non-western clients can be difficult, but is certainly not without personal and professional rewards.

\section{Commentary}

The value of this study lies not only in its particular focus on midwives' experiences of working with and caring for non-western women, and in the insights gained in relation to this client group, but also in the benefits of particular aspects of maternity care provision-some of which may be specific to the Netherlands.

The ambivalence described reflects both the challenges presented and the positive feelings associated with overcoming such challenges, and is evident in the examples chosen and language used. A key strategy described by these Dutch midwives relies on midwives being proactive and flexible, aware of cultural differences and influences-such as patients having a strong preference for physicians-and in anticipating possible practical difficulties and misunderstandings that may arise. Successful treatment also seems to involve directly facilitating contact with a range of healthcare professionals and others as needed. A need for additional time and resources, particularly in relation to interpreting services that in some instances enable considerably more effective communication with women, was also emphasised.

There are specific implications for midwifery practice and care in the Netherlands, as well as implications that apply more broadly. The findings can be used in midwifery training, particularly for those midwives likely to be working in large cities. It may, for example, be possible to use some of the material collected in creating vignettes to be used in teaching. However, these findings need to be supported and added to by qualitative and quantitative research on non-western women themselves in order to better understand their care needs, priorities and expectations in the context of maternity care. ${ }^{23}$

The authors recognised the powerful role of disadvantage and low socioeconomic status, and in many ways there are likely to be parallels in midwives' experiences of providing maternity care to women with few resources of all ethnicities and origins. This is an important point for all those working in this area to bear in mind; certainly in quantitative studies it is important to separate, where possible, the effects of disadvantage from migration status and ethnicity. ${ }^{4}$

Competing interests None.

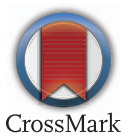

\section{References}

1. Raleigh VS, Hussey D, Seccombe I, et al. Ethnic and social inequalities in women's experience of maternity care in England: results of a national survey. $J R$ Soc Med 2010;103:188-98.

2. Henderson J, Gao H, Redshaw M. Experiencing maternity care: the care received and perceptions of women from different ethnic groups. BMC Pregnancy Childbirth 2013;13:196.

3. Jomeen J, Redshaw M. Ethnic minority women's experience of maternity services in England. Ethn Health 2013;18:280-96.

4. Alderliesten ME, Vrijkotte TG, van der Wal MF, et al. Late start of antenatal care among ethnic minorities in a large cohort of pregnant women. BJOG 2007;114: 1232-9. 